\title{
Presentation and management of depression in people with learning disability
}

\author{
Vee Prasher
}

The occurrence of depression in people with learning disability is not a late-20th century phenomenon. Reports were first published in the 1800 s describing the existence of depression in individuals with intellectual impairment (Clouston, 1883). However, as we enter a new millennium, the appropriate diagnosis, assessment and treatment of depression, and indeed all forms of mental illness in people with learning disability, will become the focus of considerable concern for all professionals working in this field. With recent national trends towards de-institutionalisation, referral of individuals with learning disability to psychiatrists to 'rule out a possible depressive illness' and to 'advise about further treatment' will increase. It remains essential for all psychiatrists in learning disability to continue to improve their diagnostic skills to better manage depressive episodes and, where possible, to learn how to prevent a future relapse. This review highlights the important presentation and management issues relating to depression in the field of learning disability. Depression in this review implies a single major depressive episode, irrespective of whether or not it conforms to any given classification system.

\section{Prevalence}

The prevalence of depression in people with learning disability has yet to be precisely determined. Reported rates to date should be accepted with caution because of a number of methodological errors, in particular: (a) confusion regarding the use of the term 'depression' - the term has been applied to 'low mood', to a single current depressive episode, to past depressive episodes and to a bipolar affective disorder;

(b) bias in sample selection - whether the sample studied accurately represents the target population;

(c) instruments with disputable validity and reliability used to determine psychopathology;

(d) appropriateness of the diagnostic criteria used; and

(e) errors in application of diagnostic criteria.

Numerous case reports have now been published reporting the presence of depression in people with learning disability.

Many of these reports have been previously reviewed (Carlson, 1979; Sovner \& Hurley 1983). Prevalence studies (Table 1) would suggest that the point prevalence rate for a major depressive illness in people with learning disability is in the order of $2-7 \%$. The rate is not dissimilar to that for the general population (3-5\%). These findings would suggest that the occurrence of depression is certainly not less common in people with learning disability than the general population, and could possibly be twice the rate. Depression can present at all ages and is reported to be more common in females than males.

There remains a paucity of information regarding: incidence rates of depression in people with learning disability; lifetime prevalence rates of depression; age and gender differences; differential rates of depression as determined by severity of learning disability; and differential rates as determined by the aetiology of the learning disability.

Vee P. Prasher is a Senior Clinical Lecturer in Developmental Psychiatry at the Department of Psychiatry, University of Birmingham, Queen Elizabeth Psychiatric Hospital, Mindelsohn Way, Edgbaston Birmingham B15 2QZ. He has a special interest in the assessment and management of mental illness in adults with learning disability. 


\section{Aetiological factors}

\section{Genetic factors}

It remains to be established whether individuals with learning disability associated with particular syndromes have a greater susceptibility for developing a depressive illness compared with other individuals with and without intellectual impairment. Individuals with Down's syndrome and fragile $X$ syndrome have been highlighted as two risk populations. Comparative studies (family studies, twin studies and studies of adoptees) investigated in the general population have, for methodological reasons, not been undertaken in populations of people with learning disabilities. The role of familial inheritance is, therefore, unknown.

\section{Organic brain disorders}

Most cases of severe learning disability have associated abnormalities in structures of the brain. Brain damage will increase difficulties in mood, behaviour, personality and language. The presence of epilepsy may further increase the risk of a person suffering an affective disorder. It is probable that underlying neurotransmitter systems are disrupted leading to a deficiency of catecholamines, and subsequently increasing the risk for depression.

\section{Coexisting physical or psychiatric disorder}

It is now well-recognised in the general population that the presence of a physical or psychiatric illness can predispose to the onset of a depressive episode. Physical morbidity is common in people with learning disability and may increase the risk of depression. Similarly, depressive symptoms can present in a person with learning disability and psychiatric illness - particularly dementia (Burt et al, 1992; Prasher, 1995a) and long-standing anxiety disorder.

\section{Social factors}

Limited information is available regarding the effects of 'ife-events' in depression in people with learning disability, but it appears that depression in this population is associated with particular social factors (Reiss \& Benson, 1985). Most people with learning disability are subject to a significant number of social stresses. These include increased health morbidity, unemployment, stigmatisation, low income, few intimate relationships and loss of social support (especially with recent de-institutionalisation). There is no definite evidence regarding a causal relationship between these factors and a depressive episode, but it is possible that, for example, resulting loneliness and feelings of helplessness are factors in the aetiology of depression. Further, people with learning disability are vulnerable to any given life-event because of their impaired capacity to withstand stress.

\section{Psychological theories}

Many psychological theories have been postulated to explain depression in the general population. For people with learning disability, there have been two principal theories. The first model, 'learned helplessness', is based on the operant theory of behaviour where individuals present with features of depression as a result of an inability to 'escape' from aversive stimuli. The second model, 'social isolation', is based on the social learning theory. Deprivation of social contact can result in behaviours analogous to clinical depression.

Table 1. Prevalence of major depression in people with learning disability

\begin{tabular}{lllll}
\hline Report & Age group & Sample size & Prevalence rate Comments \\
Collacott et al (1992) & $>16$ years & 742 & $7.8 \%^{1}$ & $\begin{array}{l}\text { Higher rate in adults with } \\
\text { Down's syndrome }\end{array}$ \\
Bouras \& Drummond (1992) & Adults & 318 & $6.6 \%$ & - \\
Meins (1993) & $>19$ years & 798 & $2.5 \%$ & - \\
Patel et al $(1993)$ & $>50$ years & 105 & $4.8 \%$ & Moderate/severe learning \\
& & & $5.0 \%$ & disability subjects \\
Prasher $(1995 b)$ & 201 & &
\end{tabular}


At present, more work is needed to investigate aetiological factors of depression in people with learning disability. Research in the field of molecular genetics will have a significant impact, when 'candidate' genes which increase the risk of depression are identified. Community care will enable researchers to investigate the social influence and social consequences of depression. The potential for prevention of depression in at least some individuals with learning disability remains to be explored.

\section{Clinical presentation}

The spectrum of symptomatology associated with depression in the general population can also occur in those with learning disabilities (Cooper \& Collacott, 1996). However, a number of clinical features are more common than others (Box 1). Cognitive aspects of depression, in particular disturbance of memory, loss of concentration and inability to think clearly, are often not reported. Further research is required to determine whether such symptoms do not generally occur in people with an underlying intellectual impairment, or whether they are present but not elicited. Recurrent thoughts of death, guilt and suicide along with perceptual changes may also be under-reported - again because of the above reasons. A number of biological changes associated with depression (psychomotor retardation, disturbed sleep, loss of appetite, loss of weight and diurnal variation in symptoms) are more readily detected. These features are often of more clinical significance than many other symptoms in people with severe and profound learning disability where there is an absence of language development. Associated suicidal behaviour in people with learning disability and depression remains rare but does occur. This is, in part, because of a limited cognitive and behavioural repertoire, the presence of physical disabilities and a lack of access to lethal means.

Although emphasis on the clinical symptomatology of depression is paramount for psychiatrists, the psychosocial aspects of depression in people with learning disability must always be considered. A number of research studies have illustrated the decline in adaptive behaviour in people with learning disability who develop a depressive illness (Prasher \& Hall, 1996).

Clinical presentation will be influenced by several factors but, in particular, the underlying severity of learning disability. Individuals with borderline and mild learning disability will present not too dissimilarly to a person without learning disability and may openly say "I feel depressed", "My life is pointless". Symptoms more marked in individuals with severe than in those with mild learning disability include psychomotor agitation, irritable mood and disturbed behaviour. Symptoms less apparent include subjective depressed mood, reduced energy and complaints of general fatigue.

\section{Assessment and diagnosis}

\section{Clinical assessment}

In clinical practice, a depressive episode will generally be diagnosed by a standard psychiatric assessment:

Box 1. Common clinical symptoms of major depression

Very common

Low mood

Irritability

Anhedonia

Decreased appetite

Change in motor activity

Sleep disturbance

Fatigue

Decreased concentration

Withdrawal

Aggression

Tearfulness

Loss of interest

Decline in social skills
Common

Weight loss

Guilt feelings

Somatic complaints

Self-injury

Destruction of property

Diurnal variation in mood

Loss of libido

Loss of confidence

Constipation

Anxiety

Obsessions/compulsions
Rare

Suicidal ideations

Change in sexual activity

Hallucinations

Delusions

Eating disorder

Hysterical conversion

Hypochondriasis 
(a) patient and informant history regarding presenting problem, psychiatric history, family history and treatment history;

(b) review of information from other professionals such as nurses, psychologists, social workers and general practitioners;

(c) mental state examination (including direct observation and self-reporting);

(d) physical examination;

(e) investigations (e.g. blood tests, electrocardiogram, electroencephalogram and brain imaging);

(f) additional written information regarding measurement of weight, sleep and behaviour by recordings made over time on standard charts;

(g) multi-disciplinary assessment (doctors, nurses, psychologists, social workers, occupational therapists and pharmacists);

(h) application of diagnostic guidelines; and

(i) trial of medication.

This remains the fundamental process of detecting a mental disorder for the vast majority of psychiatrists and, if undertaken comprehensively and correctly, will usually serve the doctor well. However, the assessment and diagnosis of depression in people with learning disability remains problematic, and it is not uncommon for a depressive illness to be difficult to detect in particular groups of individuals (Box 2).

There have been a number of developments regarding improvement in psychometric assessment instruments, principally to improve research studies, but also to improve day-to-day clinical assessments. Such instruments may improve detection, reduce diagnostic disagreement, improve treatment, allow comparisons within and between research studies, aid planning of services and aid communication between the doctor and patient/carers.

Box 2. Conditions where depression maybe masked

Patients receiving high-dose neuroleptic medication

Autism

Severe and profound learning disability

Long-standing 'acting-out' behaviours (aggression, self-injury, overactivity)

Additional medical problems - sensory impairment, epilepsy, diabetes, heart failure

Dementia

\section{Diagnostic instruments and rating scales}

Recently, instruments measuring depression specifically in people with learning disability have been proposed, for example the Psychiatric Assessment Scale for Adults with Developmental Disability (PAS-ADD; Moss et al, 1994). A number of rating scales, usually designed for the general population but modified for people with learning disability, are also available. These include the Beck Depression Inventory (Beck et al, 1961), the Zung Self-Rating Depression Scale (Zung, 1965), the Hamilton Rating Scale for Depression (Hamilton, 1960), the Mental Retardation Depression Scale (Meins, 1996) and the Self-Report Depression Questionnaire (Reynolds \& Baker, 1988). Reported correlation of such instruments with the clinical diagnosis of depression remains to be fully established, but nevertheless their utility in the assessment of depression should be noted. Instruments used in the general population to measure cognitive deficits of depression (e.g. problem-solving skills, cognitive distortions and negative statements about self) are likely to be of less significance.

As with other forms of mental illness, the detection of depression in people with learning disability is difficult for a number of reasons. These include difficulties in the understanding and expression of feelings of sadness by people with learning disability, misinterpretation of behaviours, low levels of recognition of symptoms by carers and professionals, and misdiagnosis. A high index of suspicion for depression is required by psychiatrists when assessing a person with learning disability.

Although not proven, clinicians may find that the use of such instruments along with their clinical assessment does enhance their patient management. Diagnostic accuracy can be improved, and repeat assessments using such instruments can be a means of measuring response to treatment. However, such instruments should be applied with caution. Psychiatrists prior to the application of any given instrument should satisfy themselves with the reliability, validity and the practicalities of the test.

There remain ongoing problems such as severity of learning disability, confounding medical disorders and the effects of medication on test items, rater bias and patient acquiescence (or the response tendency to agree or say 'yes').

\section{Diagnostic criteria}

There is no definitive biological test to make a diagnosis of depression. The diagnosis, therefore, is dependent on the identification and subsequent 
classification of symptoms and signs - classification being influenced by a cluster of symptoms and signs, severity, frequency, duration and pattern. Knowledge of the underlying cause is not a prerequisite. A number of studies have investigated the role of standard diagnostic criteria and their applicability to people with learning disability. The consensus view from such studies is that for mild or moderate learning disability, DSM-III-R criteria (American Psychiatric Association, 1987) or ICD-10 criteria (World Health Organization, 1992) are applicable to this population. Difficulties arise for people with severe and profound learning disability where standard diagnostic criteria require modification to emphasise the biological features and behavioural changes of depression. Such diagnostic criteria will need to take into account traits associated with severe learning disability or with a possible behavioural phenotype. Further research is required.

\section{Investigations}

Laboratory investigations should include routine haematological, biochemical and endocrine screening to exclude a physical disorder presenting as a depressive episode, in particular, exclusion of thyroid dysfunction, diabetes and anticonvulsant toxicity.

Specific tests include the dexamethasone suppression test (DST) proposed for the diagnosis of depression in the general population. This is based on the assumption that there is a disturbance of the hypothalamic-pituitary-adrenal axis in depressive illness - patients with depression do not suppress cortisol production on administration of dexamethasone. Contradictory research results are reported in studies of adults with learning disability and depression (Wolkowitz, 1990, Mudford et al. 1995). The validity of the DST remains in doubt and caution is required in its use to aid the diagnosis of depression in people with learning disability.

In the general population, a number of other tests have shown abnormalities which correlate with the clinical diagnosis of depression. These include changes in the sleep or waking electroencephalogram, impaired evoked potential response and abnormalities in functional and structural neuroimaging of the brain. Such tests remain areas of future research and, as yet, are of limited value in people with learning disability who become depressed.

\section{Differential diagnoses}

As previously discussed, the accurate diagnosis of a depressive episode in a person with learning disability can be difficult. The differentiation, therefore, from other conditions can be even more problematic (Box 3).

People with learning disability have increased physical morbidity compared with the general population. Distinguishing a depressive disorder from a mood disorder due to a general medical illness (e.g. vitamin deficiency, hypo-/ hyperthyroidism or head injury) should always be considered. If a physical illness is present, clinical features usually do not reach full diagnostic criteria for a depressive disorder. Evidence from the history, physical examination or laboratory tests may highlight that the mood disturbance is secondary to an underlying physiological disorder.

Drug-induced mood disorder is not uncommon in people with learning disability. This results from a direct physiological effect of the medication or can occur as part of drug toxicity or drug withdrawal. Depressive symptomatology is present, but again, often does not fulfil recognised diagnostic criteria for a depressive disorder. The type of medication, dosage, onset and course of the mood disorder can aid differentiation of a drug-induced mood disorder from a major depressive illness. Particular drugs of concern are anticonvulsants, hypnotics, antipsychotics, analgesics, anticholinergics, oral contraceptives and steroids.

If particular symptoms characteristic of a depressive illness (low mood, episodes of crying, loss of appetite, insomnia and loss of weight) result as part of a reaction to the death of someone emotionally close, then a bereavement reaction should be considered. The time of onset, duration and severity may vary widely, making an accurate diagnosis difficult.

Many clinical features of depression are seen in anxiety disorders, and vice versa. Discrimination by clinical presentation or by treatment response may prove to be of limited help. Research of neurotic disorders in people with learning disability is

Box 3. Common differential disorders for a depressive episode

General medical illness (endocrine disorder, metabolic disorder, neurological conditions, infections, cancer)

Medication effects (including toxicity and withdrawal effects)

Bereavement reaction

Anxiety states

Dementia

Behavioural disorder 
lacking - but 'situational anxiety' would be more suggestive of an anxiety disorder, whereas as 'persistent depressed mood' would be more supportive of a depression illness.

The occurrence of depressive symptoms in dementia of the Alzheimer's type and the differential diagnosis between dementia and depression has been highlighted for adults with Down's syndrome (Burt et al, 1992; Prasher, 1995a). Patients with depression may present with marked cognitive defects (pseudodementia). However, fluctuating mood, an anxious rather than a blunting affect, patchy cognitive impairment and prominent biological features of depression would suggest a depressive episode rather than a dementing process. Response to treatment and longitudinal review can often clarify the situation.

\section{Treatment}

The general approach to the appropriate management and treatment of depression in people with learning disability is not too dissimilar to that for the general population. Treatment must follow a detailed assessment which should include longitudinal information and clinical features consistent with diagnostic criteria.

\section{Drug therapy}

The mainstay of treatment is antidepressant drug therapy - this may be :

(a) tricyclic antidepressants (TCAs, e.g. amitriptyline, lofepramine, dothiepin)

(b) monoamine oxidase inhibitors (MAOIs, e.g. phenelzine)

(c) selective serotonin reuptake inhibitors (SSRIs, e.g. fluoxetine, fluvoxamine, paroxetine and sertraline)

(d) noradrenergic and specific serotonergic antidepressants (NaSSAs, e.g. mirtazapine)

(e) selective noradrenaline reuptake inhibitors (NARIs, e.g. reboxetine)

(f) serotonin/noradrenaline reuptake inhibitors (SNRIs, e.g. venlafaxine)

For people with learning disability, more so than for the general population, the rational use of antidepressants is dependent on the awareness of their side-effects, drug interactions and secondary complications. Tricyclic medication, for example, should not be used in individuals with cardiac disease or dementia where the risk of experiencing detrimental side-effects is high. Psychiatrists in the field of learning disability need to be extra vigilant because of the lack of self-reporting of side-effects in people with limited verbal communication skills.

The SSRIs are thought to be as efficacious as the older forms of antidepressants, but the side-effect profile (less sedation, less cardiotoxicity and fewer anticholinergic side-effects) is more benign and the risk from overdose much lower. SSRIs may have greater efficacy in subjects with self-injurious and aggressive behaviour.

If there is no benefit with a five- to eight-week course of an antidepressant, including at a higher dosage, the diagnosis should be reviewed. An alternative antidepressant can be prescribed but an MAOI should be used with caution - and only after 10 days after stopping the tricyclic and only when individuals and carers are fully aware of serious interactions with alcohol, cheese and other prohibited foods. Lithium carbonate can be added to a tricyclic to augment the action of the antidepressant.

Discontinuation reactions (withdrawal symptoms) have been reported for virtually all classes of antidepressants. Reactions usually start two to three days after stopping medication, are reversible with recommencement of therapy and are mostly mild and short-lived. Common symptoms include dizziness, nausea, headache, lethargy, sleep disturbance, agitation and restlessness. The likelihood of a discontinuation reaction can be prevented by gradual reduction of the antidepressant over four to six weeks. Standard precautions must be undertaken in the field of learning disability in terms of lowest dosage possible, slow increase, close monitoring for side-effects and regular review of medication.

\section{Psychotherapy and behavioural therapy}

For people with mild learning disability, psychotherapy and behavioural therapy may enable underlying psychological factors to be explored. This applies in particular to individuals presenting with self-harm/suicidal behaviour. Such treatments can be used along with a course of antidepressants. Antidepressants can often lift mood to allow a person to work more effectively in the other treatments. To date, limited research is available, but case reports have been published demonstrating the effectiveness of non-pharmaceutical therapy for adults with learning disability and depression.

\section{Electroconvulsive therapy}

Electroconvulsive therapy (ECT) for people with learning disability is extremely uncommon. Other 
than case reports reporting benefit, the author is not aware of any systematic review of the effectiveness of ECT for people with learning disability and depression. The indications should not be too dissimilar to those for the general population - severe illness with significantly impaired food and fluid intake, psychotic depression resistant to antidepressants, previous response to ECT and pharmacotherapy contraindicated. As in the general population, it is likely that a good response is expected in depressive episodes where delusions, hallucinations and biological features are present. Maintenance drug treatment is required to prevent relapse soon after treatment.

\section{Social support}

Professional intervention for the assessment and appropriate management of a depressive episode is important. However, carers and non-health professionals have a significant role to play. A simple and understandable explanation of the illness to the patient and their carers can aid cooperation. Support to patients and carers by a community nurse can improve monitoring of the illness in the community. Increased physical activity at home or at their day placement can reduce feelings of tiredness and apathy. Maintaining the daily routine and contact with friends can allay feelings of helplessness. The encouragement of activities which the patient has previously enjoyed can contribute to improving selfesteem. Carers should gently continue to encourage eating and maintenance of appetite and weight.

\section{Prognosis}

There have been limited studies looking at the prognosis of a depressive episode in people with learning disability. The short-term outlook for a single depressive episode varies from full recovery to ongoing difficulties with features suggestive of a chronic depressive disorder, characterised by partial recovery of functional capacity (Prasher \& Hall, 1996). Future relapse is not uncommon. The course of a depressive illness will be dependent on whether it is part of a bipolar or unipolar disorder, age of onset, and effectiveness of treatment. Limited information is available regarding the long-term followup of depression in people with learning disability.

Further research involving treatment trials is required regarding benefits of maintenance therapy (antidepressants, lithium and carbamazepine) to prevent future depressive illnesses. Limited information suggests that people with learning disability, as with the general population, can benefit from maintenance therapy. Other important areas of interest regarding depression in people with learning disability are: (a) the age effects on the severity, duration and treatment response; (b) probability of recovery; (c) likelihood of relapse; and (d) rates of in-patient care.

\section{Conclusions}

It remains imperative that all psychiatrists caring for people with learning disability continue to improve their clinical skills in the assessment and treatment of mental disorders. Depression is relatively common with a prevalence similar to that in the general population. Whether or not underlying genetic abnormalities or intellectual impairment predispose to depression requires further investigation. Particular risk groups appear to be people with Down's syndrome and fragile $X$ syndrome. With systematic assessment and the use of appropriate instruments, accurate diagnosis of a depressive episode is possible. Subsequent management usually involves a course of antidepressant therapy along with non-pharmaceutical intervention. There remain several areas of future research, the findings of which should improve the provision of clinical care.

\section{References}

American Psychiatric Association (1987) Diagnostic and Statistical Manual of Mental Disorders (3rd edn, revised) (DSM-III-R). Washington, DC: APA.

Beck, A. T., Ward, C. H., Mendelson, M., et al (1961) An inventory for depression. Archives of General Psychiatry, 4, 561-585.

Bouras, N. \& Drummond, C. (1992) Behaviour and psychiatric disorders of people with mental handicaps living in the community. Journal of Intellectual Disability Research, 36, 349-357.

Burt, D. B., Loveland, K. A. \& Lewis, K. R. (1992) Depression and the onset of dementia in adults with mental retardation. American Journal on Mental Retardation, 96, 502-511.

Carlson, G. (1979) Affective psychoses in mental retardates. Psychiatric Clinics of North America, 2, 499-510.

Clouston, T. S. (1883) Clinical Lectures on Mental Diseases. London: Churchill.

Collacott, R. A., Cooper, S.-A. \& McGrother, C. (1992) Differential rates of psychiatric disorders in adults with Down's syndrome compared with other mentally handicapped adults. British Journal of Psychiatry, 161, 671-674.

Cooper, S. A. \& Collacott, R. A. (1996). Depressive episodes in adults with learning disabilities. Irish Journal of Psychological Medicine, 13, 105-113.

Hamilton, M. (1960) A rating scale for depression. Journal of Neurology, Neurosurgery and Psychiatry, 23, 56-62.

Meins, W. (1993) Prevalence and risk factors for depressive disorders in adults with intellectual disability. Australia and New Zealand Journal of Developmental Disabilities, 18, 147-156. 
- (1996) A new depression scale designed for use with adults with mental retardation. Journal of Intellectual Disability Research, 40, 222-226.

Moss, S. C., Ibbotson, B. \& Prosser, H. (1994) The Psychiatric Assessment Schedule for Adults with Developmental Disability (The PAS-ADD); Interview Development and Compilation of the Clinical Glossary. Manchester: Hester Adrian Research Centre, University of Manchester.

Mudford, O. C., Barrera, F. J., Murray, A., et al (1995) The dexamethasone suppression test and the diagnosis of depression in adults with severe and profound developmental disabilities. Journal of Intellectual Disability Research, 39, 275-283.

Patel, P., Goldberg, D. \& Moss, S. (1993) Psychiatric morbidity in older people with moderate and severe learning disability II: The Prevalence Study. British Journal of Psychiatry, 163, 481-491.

Prasher, V. P. (1995a) Age-specific prevalence, thyroid dysfunction and depressive symptomatology in adults with Down syndrome and dementia. International Journal of Geriatric Psychiatry, 10, 25-31.

- (1995b) Prevalence of psychiatric disorders in adults with Down's syndrome. European Journal of Psychiatry, 9, 77-82.

- \& Hall, W. (1996) Short-term prognosis of depression in adults with Down's syndrome: association with thyroid status and effects on adaptive behaviour. Journal of Intellectual Disability Research, 40, 32-38.

Reiss, S. \& Benson, B. A. (1985) Psychosocial correlates of depression in mentally retarded adults: I. Minimal social support and stigmatization. American Journal of Mental Deficiency, 89, 331-337.

Reynolds, W. M. \& Baker, J. A. (1988) Assessment of depression in persons with mental retardation. American Journal on Mental Retardation, 93, 93-103.

Sovner, R. \& Hurley, D. N. (1983) Do the mentally retarded suffer from affective illness? Archives in General Psychiatry, 40, 61-67.

Wolkowitz, O. M. (1990) Use of the dexamethasone suppression test with mentally retarded persons: review and recommendations. American Journal on Mental Retardation, 94, 509-514.

World Health Organization (1992) The ICD-10 Classification of Mental and Behavioural Disorders. Clinical Descriptions and Diagnostic Guidelines. Geneva: WHO.

Zung, W. W. K. (1965) A self rating depression scale. Archives of General Psychiatry, 12, 63-70.

\section{Multiple choice questions}

1. The prevalence of major depression in people with learning disability is:

a three times higher in people with mild learning disability compared with those with severe learning disability

b $30 \%$

c probably higher in people with Down's syndrome when compared with people with learning disability of unknown cause

d $5 \%$

e much less common than in people of average ability.
2. People with learning disability suffer from depression because:

a a specific gene has been located on chromosome 6

b organic brain pathology may be a predisposing factor in some people

c they lack capacity to withstand stress

d there is usually a strong family history of depression

e adverse experiences during development (e.g. institutional care or abuse) may be predisposing factors in some people.

3. Clinical features of depression in people with learning disability include:

a a high rate of suicide

$b$ an increase in aggression

c the dexamethasone suppression test is a definitive test

d a differential diagnosis of dementia

e an irritable mood, sometimes in the absence of miserable mood.

4. In planning treatment:
a a multi-disciplinary assessment is pointless
$b$ rating scales have a role to play
c the patient's previous experiences may of value
d carer views add little to the patient's account
e vigilance is required in evaluating drug side- effects in those who have limited verbal communication skills.

5. People with learning disability and depression:
a often respond to antidepressants
b always require in-patient care
c should never be treated with ECT
$\mathrm{d}$ will invariably fully recover
e may benefit from psychological and social treatments in addition to drug treatment.

MCQ answers

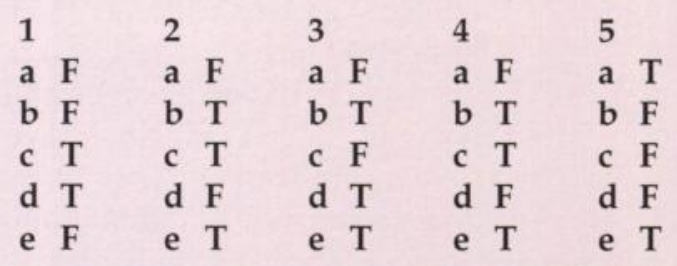

